
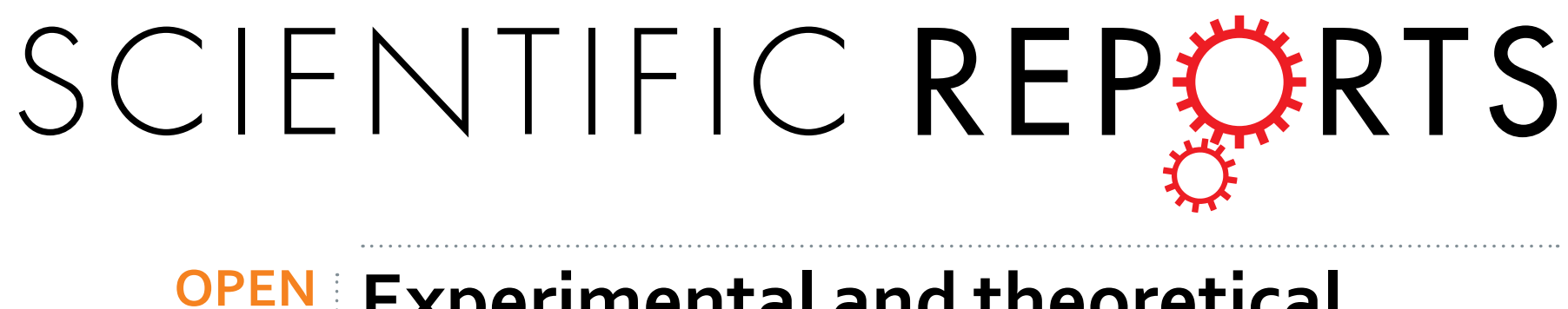

\title{
Experimental and theoretical
} studies of nonlinear dependence of the internal resistance and

Received: 10 January 2017

Accepted: 07 March 2017

Published: 05 April 2017

\section{electrode thickness for high performance supercapacitor}

Xilong Liu ${ }^{1}$, Xiaohang Dai ${ }^{1}$, Guodong Wei ${ }^{1}$, Yunlong Xi ${ }^{1}$, Mingjun Pang ${ }^{2}$, Volodymyr Izotov ${ }^{1}$, Nickolai Klyui ${ }^{1,3}$, Dmytro Havrykov ${ }^{1,3}$, Yuan $\mathrm{Ji}^{1}$, Qing Guo ${ }^{1}$ \& Wei Han ${ }^{1}$

In this study, the internal resistance with the increasing of electrode thickness in a typical nanoporous carbon-based supercapacitor and their corresponding electrochemical performances were designed and investigated in detail. As for the carbon-based double electrode layer electrochemical system, electrochemical experiments greatly support the fact of nonlinear dependence and indicate that the curve of internal resistance vs. electrode thickness can have a minimum value when the thickness increasing from 10 to $140 \mu \mathrm{m}$. To explain the underlying mechanisms responsible for the nonlinear dependence, a theoretical model based on a porous electrode/electrolyte electrochemical system was proposed. As expected, the results of calculations carried out in the framework of the proposed model can be very good agreement with the experimental data. According to the calculation, the optimized electrode thickness is $53.1 \mu \mathrm{m}$ corresponding to the minimum value of $S C$ internal resistance. Obviously, the current research results might greatly support the nonlinear conclusion instead of linear relationship between the internal resistance and the electrode thickness and may shed some light on the fabrication and exploration of supercapacitors with high power density.

An intense development of high performance electric vehicles (especially electric cars) requires novel rechargeable power sources that could endure up to one million charging/discharging cycles as well as high specific power and high specific energy ${ }^{1}$. Up to date, though several kinds of energy storage devices have been developed for the hybrid electric, it is widely accepted that the electric double-layer capacitors, also known as the supercapacitors (SC) is an ideal choice due to the long durable life with practically unlimited charging/discharging cycles $^{2}$. A typical SC device can be usually consisted of two carbon-based electrodes that are electrolyte-impregnated and separated with a separator, whose structure is similar with that of a common electrolytic capacitor. Yet different from the electrodes of the common electrolytic capacitors, a SC electrode consists of a metal collector coated with a layer of nanoporous carbon material with high specific surface $\operatorname{area}^{2}$.

A key parameter to determine the SC performance is the specific energy density, and for this reason, there is considerable interest in enhancing their values by optimizing SC electrode structure and design. The specific energy density for commercial SC devices can reach up to $6 \mathrm{~W} \cdot \mathrm{h} / \mathrm{kg}$ from the key firms' products ${ }^{3}$. It is also well known that specific energy value can be mostly related to the fabricated SC electrodes. According to the energy store mechanism, in the course of SC charging, the energy can be accumulation of electric charge in the double layer formed at the carbon electrode/electrolyte interface ${ }^{2}$. Therefore, the specific capacity (based on the energy density formula $\mathrm{E}=0.5 \mathrm{CV}^{2}$, in fact, the energy density is only related to the specific capacity if the voltage is fixed at a certain value) for a typical carbon-based SC with organic electrolyte can be about $10 \mu \mathrm{F} / \mathrm{cm}^{2-4}$. The specific

${ }^{1}$ Key Laboratory of Physics and Technology for Advanced Batteries, Ministry of Education, College of Physics, Jilin University, Changchun 130012, China. ${ }^{2}$ Institute of Carbon Materials Science, Shanxi Datong University, Datong 037009, China. ${ }^{3}$ V. Lashkaryov Institute of Semiconductor Physics, National Academy of Sciences of Ukraine, 41 Nauki Pr., 03028 Kyiv, Ukraine. Correspondence and requests for materials should be addressed to V.I. (email: vizotov@bigmir.net) or Q.G. (email: qingguo@jlu.edu.cn) orW.H. (email: whan@jlu.edu.cn) 
capacity can be influenced by the parameters of the conductivity, surface area and pore size distribution of carbon material, binder content, electrode thickness, and et al. Among these parameters, large specific surface area (over $1000 \mathrm{~m}^{2} / \mathrm{g}$ ) of porous carbon material used in the SC electrodes can facilitate the achievement of high specific energy density in the final SC device.

Another important parameter to determine the SC performance is the internal resistance $\left(R_{\text {in }}\right)$. The $R_{\text {in }}$ value determines another important SC parameter that generally called power density, namely, its specific pulse power $P(\mathrm{~W} / \mathrm{kg})$ that can be calculated from the following equation:

$$
P=\frac{U^{2}}{4 M R_{\text {in }}},
$$

where $U(\mathrm{~V})$ and $M(\mathrm{~kg})$ are the SC operating voltage and mass, respectively. For products from the leading manufacturers, $P$ value ranges from $10 \mathrm{~kW} / \mathrm{kg}$ to $20 \mathrm{~kW} / \mathrm{kg}^{3}$. Based on Eq. (1), higher $P$ values can be achieved by decreasing the $\mathrm{R}_{\text {in }}$ value. According to the reports in the literature, the $\mathrm{R}_{\text {in }}$ depends on the following factors: (i) the contact resistance between the metal Al current collector and the electrode component made of a porous carbon material; (ii) resistance resulted from the interelectrode region; (iii) resistance from the electrode component and soaked with electrolyte. From here on, such the electrode components will be referred to as the energy-storage electrode components.

It is widely accepted that the main contribution to the $\mathrm{SC} \mathrm{R}_{\mathrm{in}}$ comes from the resistance of the energy-storage electrode component. According to reports, it can depend on many factors: (i) size of pores of the activated carbon material, (ii) composition of the material used for production of the energy-storage electrode component, (iii) electrolyte composition, and (iv) thickness of the energy-storage electrode component. To reduce the SC $\mathrm{R}_{\text {in }}$ value, several cost-effective and simple methods were explored for solving this challenge from the industry technological views ${ }^{5-8}$. Though decades of sustained efforts, it is still a very hot study field and still need substantial progress on developing new technologies for reducing the $\mathrm{R}_{\mathrm{in}}$. Among these mentioned factors, the resistance resulted from the thickness of the energy-storage electrode component can have a main influence on the SC performance for the industry products. For the purpose of practical application, high mass loading of active materials with thick electrode for the fabrication of supercapacitors can achieve high energy density. However, the increase in the electrode thickness usually leads to increasing resistance as well as reducing specific capacitance and energy density, due to close-packed structures resulting in a limited electrochemically active surface area. Moreover, the resistance dependence of electrode thickness is not clearly and even inconsistent in the literature reports. Some reports support this dependence is linear ${ }^{9-11}$, while other ones believe that the functional dependence of SC internal resistance on electrode thickness is nonlinear ${ }^{12,13}$. Therefore, experiment and theory study should be performed to discover the underlying mechanism to increase the utilization efficiency of electroactive material at large mass loading with optimized electrode thickness. In addition, application of the electric-spark method for processing of a metal current collector by implanting carbon particles into its surface leads to considerable decrease of contact resistance between the current collector and energy-storage electrode component, which could be regarded as a promising method for practical application. The detailed study of the theory and performance of the current collector modification could be found in our group report in the near future.

In this study, the resistance dependence of the electrode thickness and their corresponding electrochemistry performance were investigated in detail. The nonlinear results for $S C \mathrm{R}_{\mathrm{in}}$ dependent of electrode thickness in a typical carbon-based two double layer SC can effectively eliminate the arguments ${ }^{9-13}$. More importantly, a theory model based on electrochemical system "porous carbon material/organic electrolyte" was proposed for explaining the nonlinear dependence fact. By tuning the electrode thickness alone over a wide range from 10 to $140 \mu \mathrm{m}$ in the course of experiments (the rest of system parameters remaining constant), it is found that the theory calculations can agree well with the experimental results.

\section{Methods}

Raw materials. All the reagents such as 1-Methyl-2-pyrrolidinone (NMP), carbon super P, and polyvinylidene fluoride (PVDF) used in this study were of analytical grade and were used as received without any further purification The used activated carbon powders were purchased from "SHIHEZI SETDTIANFU TECHNICAL" (China) and before use, let them pass through a sieve with pore diameter of $10 \mu \mathrm{m}$.

Preparation of the electrodes. The electrode slurry for preparation of energy-storage electrodes were using a mixed suspension solution containing with NMP (solvent), activated carbon powders, Carbon Super P as a conducting additive, PVDF served as a binder, followed mixing these materials by means of ultrasonic treatment. The weight ratio between NMP, activated carbon material, conductive additive and PVDF was fixed at 75: 22.5: 1.25: 1.25. Subsequently, the electrodes were made by deposition of suspension layers using a "Doctor Blade" installation onto a modified aluminum foil. The electrodes were then dried for $72 \mathrm{~h}$ in a vacuum oven (pressure of $1 \mathrm{kPa}$ ) at a temperature of $150^{\circ} \mathrm{C}$ to remove NMP from the carbon-bearing electrode components.

Structure characterizations. The activated carbon powders used in this research were characterized with field emission scanning electron microscopy (FESEM, S-4800, Hitachi, Japan), X-ray diffraction (XRD, D8 ADVANCE, Bruker, German) and Transmission electron microscope (TEM, JEM-2100F, JEOL, Japan). The pore size distribution for that powder was determined using nitrogen adsorption-desorption at a temperature of $77 \mathrm{~K}$ on a specific surface area and porosity analyzer (ASAP 2020HD88, Micromeritics, USA).

Electrochemical measurements. The electrochemical characteristics of the electrodes were examined using typical SC device models containing 1.3 $\mathrm{M}$ of $\mathrm{Et}_{4} \mathrm{NBF}_{4}$ solution in acetonitrile as the electrolyte. Cyclic 
voltammeter $(\mathrm{CV})$ tests were performed at the applied voltages of 0-2.5 V, Galvanostatic charge-discharge (GCD) tests were performed at $100 \mathrm{~mA}$, and impedance spectroscopy in the frequency range from $10 \mathrm{kHz}$ down to $0.01 \mathrm{~Hz}$ by using a bipotentiostat (760E, China). All the electrochemical tests were performed at room temperature.

\section{Results}

As shown in Fig. 1a, according to the Barrett-Joyner-Halenda (BJH) pore size distribution analysis determined from the adsorption branches, the as-used activated carbon powders contain micro-, meso- and macropores with a BET surface area of $\sim 1900 \mathrm{~m}^{2} / \mathrm{g}$. From the inset in Fig. 1a, these pores are most micro- and mesopores with the diameter up to $3 \mathrm{~nm}$, which mainly contribute to the surface area value. Figure $1 \mathrm{~b}$ presents the morphology and microstructures of the used activated carbon powders. The powders are consisted of irregular particles with the size up to $10 \mu \mathrm{m}$. Figure $1 \mathrm{c}$ is the XRD pattern of used porous carbon powder, indicating the amorphous nature. From the HRTEM image as displayed in Fig. 1d, the mesoporous of the activated carbon can be clearly observed with the pore size distibution around $2 \mathrm{~nm}$. When used for fabricating electrode, these particles can be combined to form paper-like electrode sheet with desired thickness by the binder. The biomass-derived activated carbon powders are very loosely packed and contained many micro- and mesopores in a single particle body. Obviously, micro- and mesopores with size less than $3 \mathrm{~nm}$ can contribute a significant portion of pore volume in the particle according to our bet measurements. Due to smaller micro pores $(<2 \mathrm{~nm})$ are more difficult to access during the SC charging-discharging cycles, these pores $(>2 \mathrm{~nm})$ can serve as transport channels that pass from the metal collector through the energy-storage electrode component to the electrode surface. The electrodes were prepared by coating slurry with different thicknesses varied from $14 \mu \mathrm{m}$ to $133 \mu \mathrm{m}$ using a "Doctor Blade" installation onto the aluminum foil (size: $3 \mathrm{~cm}^{\star} 3 \mathrm{~cm}$ ) modified by the electric-spark method ${ }^{8}$ (Fig. 1e). The electrode together with $\mathrm{Al}$ foil (Fig. 1f) was dried for $72 \mathrm{~h}$ in a vacuum oven (pressure of $1 \mathrm{kPa}$ ) at the temperature of $150{ }^{\circ} \mathrm{C}$ to remove NMP from the carbon-bearing electrode component. Subsequently, the resulting electrodes were placed into a glovebox under argon atmosphere. The SC device models can be assembled with two electrodes with the same coating thickness and the two electrodes are parted with a separator produced by Nippon Kodoshi Corporation (Japan). After the electrodes and separator were together loaded into a case of $130 \mu \mathrm{m}$ thick laminated aluminum foil produced by Showa Denko K.K. (Japan), the case was sealed over along its perimeter with a special Showa Denko hot-melt glue. A polypropylene connecting pipe was inserted into a hot-melt glue layer in the course of sealing. After sealing process, $1.3 \mathrm{M}$ of $\mathrm{Et}_{4} \mathrm{NBF}_{4}$ solution in acetonitrile (as electrolyte) was injected into the SC models through the connecting pipe. Finally, the SC device models (Fig. 1g) were fabricated after the connecting pipe was taken away.

Electrochemical performances of the SC models with the electrode thickness in the range of $10-140 \mu \mathrm{m}$ were studied by using CV within a $0.0-2.5 \mathrm{~V}$ range at potential scanning speed of $5 \mathrm{mV} / \mathrm{s}$ and GCD with $100 \mathrm{~mA}$ current, impedance spectroscopy was performed with the frequency range from $10 \mathrm{kHz}$ down to $0.01 \mathrm{~Hz}$, respectively. All the electrochemical measurements were performed at room temperature. As shown in Fig. 2a, the shape of all the CV profiles is nearly the ideal rectangular shapes, indicative of highly capacitive behavior with good ion response. No obvious current density peaks and drastic growth in all the measured CV curves, indicating no pseudo capacitive capacitance contribution and absence of unwanted electrochemically active impurities in that potential range. The form of ideal rectangular $\mathrm{CV}$ curves enables one to state that only adsorption and desorption of cations $\mathrm{Et}_{4} \mathrm{~N}^{+}$and anions $\mathrm{BF}_{4}{ }^{-}$occur at the electrode surface in SC models over a given potential range. The SC parameters can be determined using impedance spectroscopy displayed in Fig. $2 \mathrm{~b}$. The $\mathrm{R}_{\mathrm{in}}$ of SC model that corresponded to the position of experimental point on the real $Z^{\prime}$ axis of the impedance curve can be determined at the maximal frequency of $10 \mathrm{kHz}$. Therefore, the $\mathrm{R}_{\mathrm{in}}$ measurement values with changed electrode thickness can be obtained presented in Fig. 2c.

\section{Discussion}

Obviously, from Fig. 2c the dependence of internal resistance on electrode thickness is nonlinear instead of linear for the electrochemical system under investigation. Therefore, a possible mechanism of electrochemical processes proceeding in SC should explain the above nonlinear dependence fact. As stated above, the energy-storage electrode component consists of porous activated carbon structures with rich of micro-, meso- and macropores and these pores with the surface area of $\sim 1900 \mathrm{~m}^{2} / \mathrm{g}$ can be serves as transport channels for electrolyte ions. When charging the SC, the electrolyte ions can penetrate into the energy-storage electrode component through these transport channels and can be adsorbed on the pore surface of carbon material particles. In the course of SC discharging, the desorbed ions can diffuse away from the surface adsorbed sites of the energy-storage electrode component. The diffuse journey for desorbed ions can be divided as followed steps (shown in Fig. 3): (1) Firstly, the ions can diffuse through pores of the carbon material into the transport channels. The charge induced by ions at the carbon material surface can move parallel to the positive ion diffusion. (2) At the second stage, the ions can move through the transport channels towards the electrode surface and at the same time the induced charge can move parallel to the positive ion movement. (3) At the final stage, the ions enter into the electrolyte bulk, while the induced charge goes through the energy-storage electrode component to the metal collector and then comes to the external electrical circuit.

To build a model for description of the above mechanism of SC discharging, an assumption that the pores of activated carbon material as well as transport channels are uniformly distributed over the whole volume of the energy-storage electrode component. The total thickness of the energy-storage part of electrode as the model is $h$. The model parameters can be determined from the experimental data and all the parameters can be calculated by using the ideal single layer electrode sheet with per $1 \mathrm{~cm}^{2}$ of visible surface. Resistivity of the transport channels and the energy-storage electrode component can be determined from the piece of experimental curve $\mathrm{R}_{\text {in }}(\mathrm{h})$. The dependence of internal resistance on $h$ is described by the straight-line equation: 
a
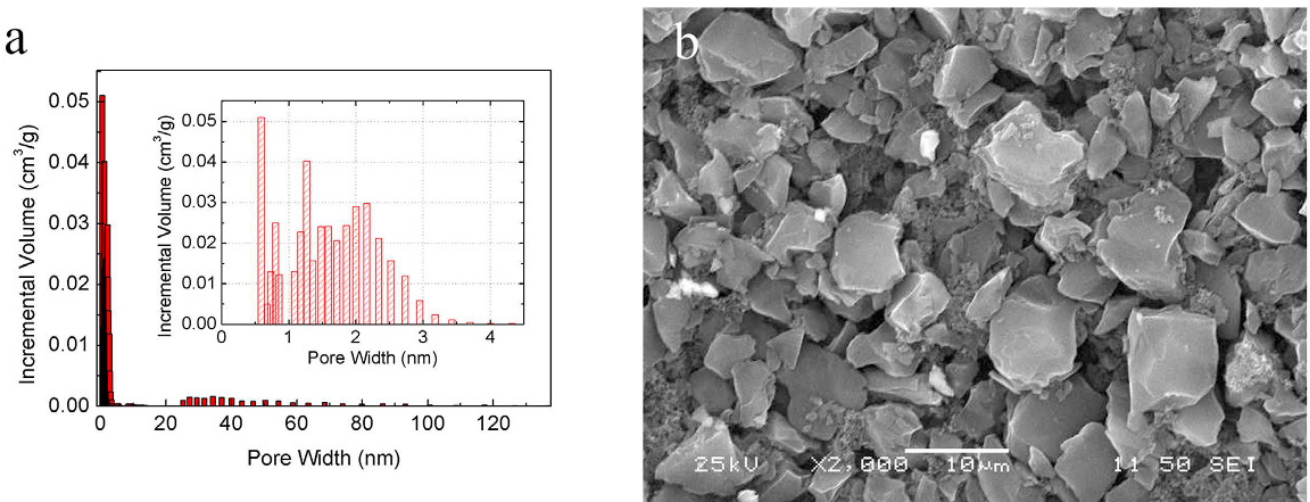

$\mathrm{C}$
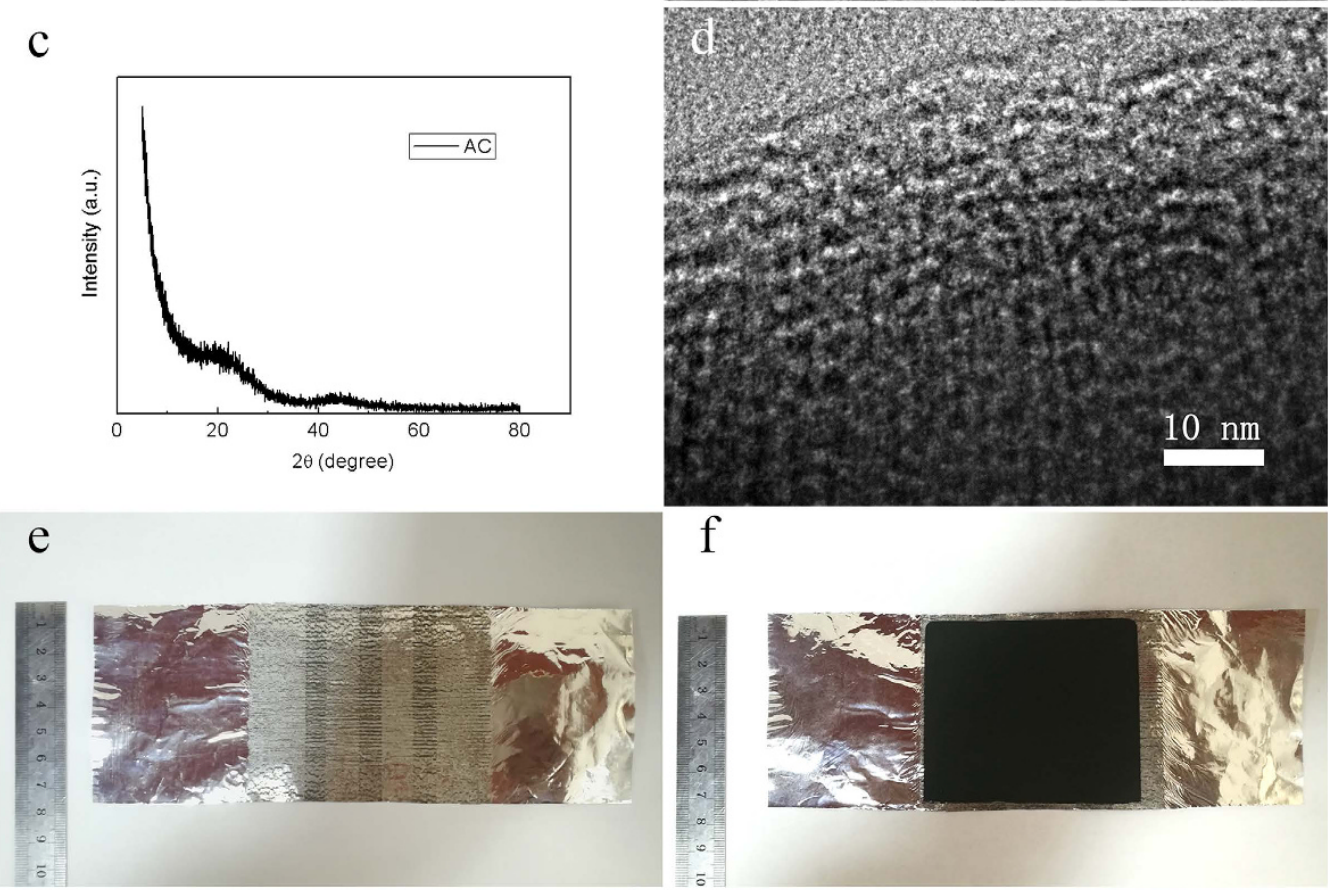

$\mathrm{f}$
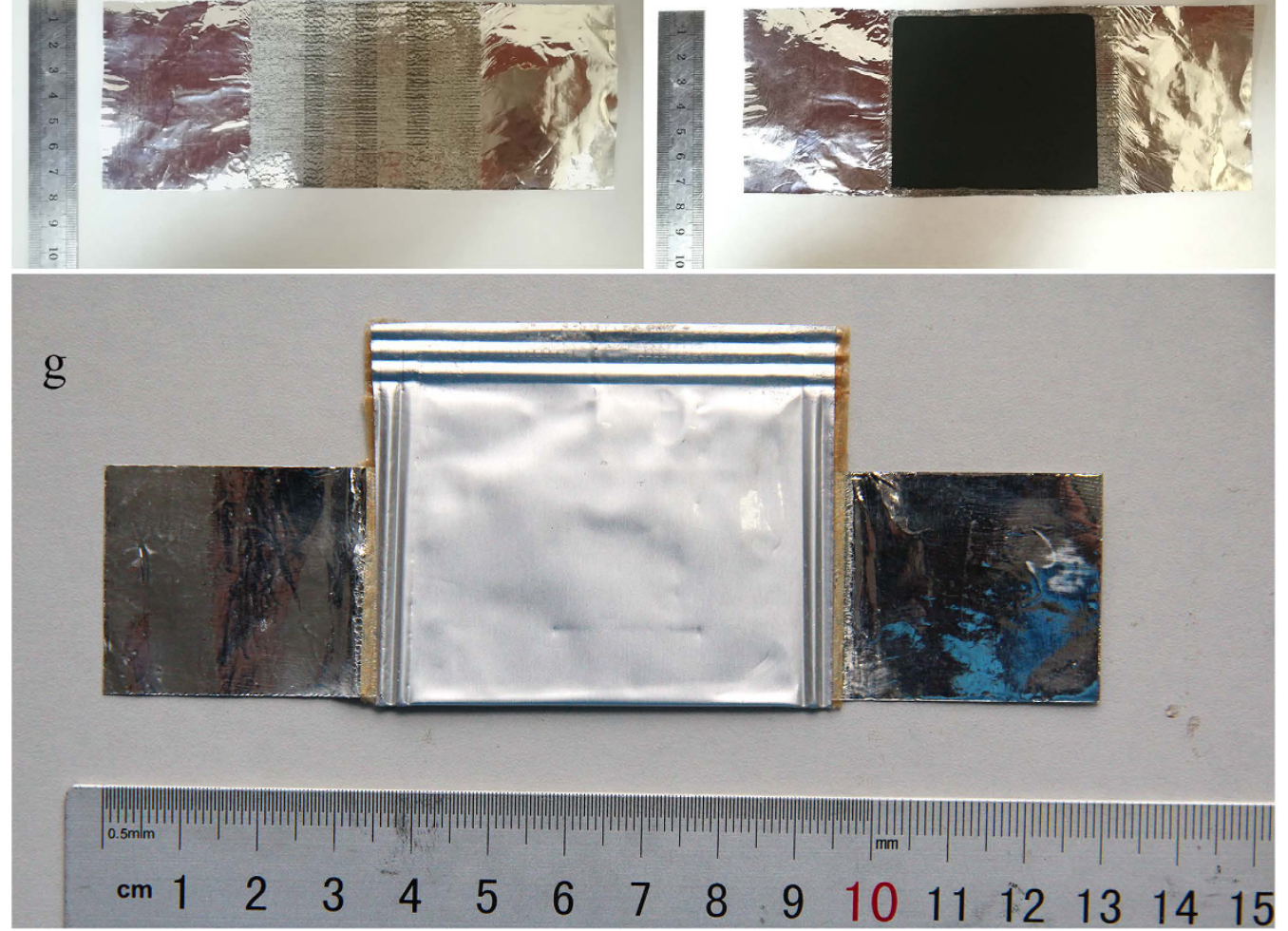

Figure 1. (a) The pore size distribution and (b) a typical SEM image of activated carbon powders, respectively. The inset in (a) is the pore size distribution curve with enlarging the X-axis in the range of $0-4 \mathrm{~nm}$. (c) XRD pattern of porous carbon powder and (d) the mesoporous on the activated carbon powders surface, respectively. Photo images of (e) aluminum foil modified by the electric-spark method, (f) the electrode sheet with coating electrode slurry, and (g) the final sealed SC device model, respectively. 
a

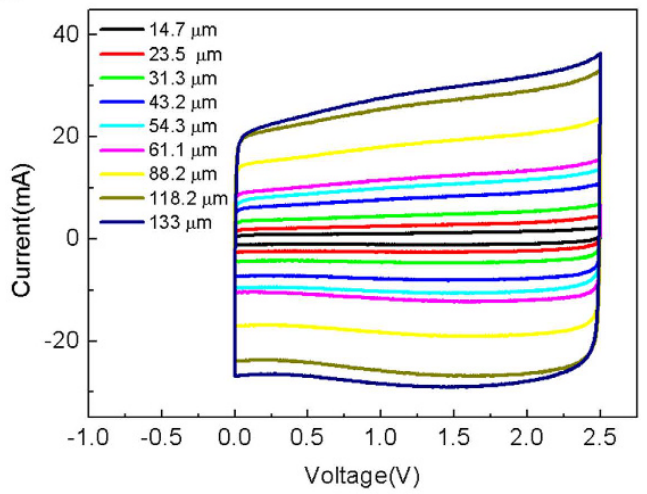

C

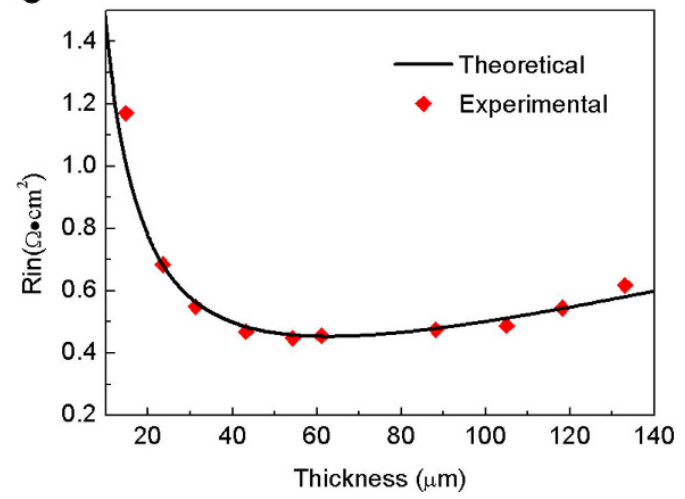

b

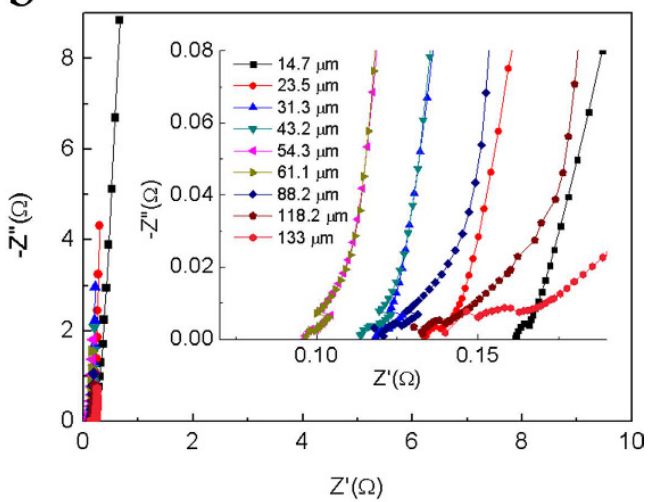

d

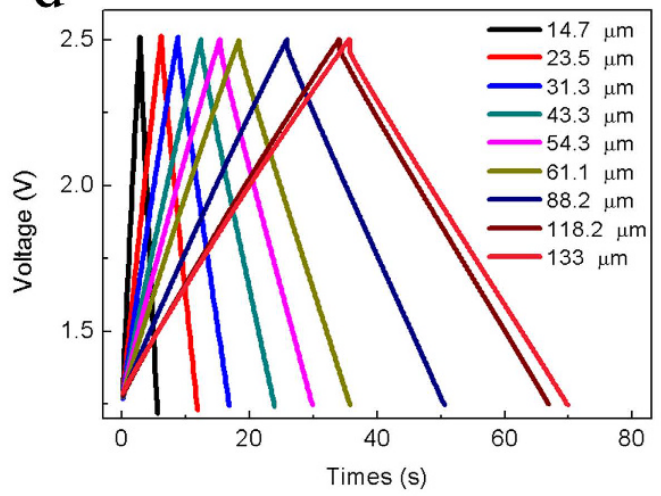

Figure 2. (a) CV curves measured at the potential scanning speed of $5 \mathrm{mV} / \mathrm{s}$ for the $\mathrm{SC}$ models and (b) their corresponding impedance hodographs for SC models with different electrode thicknesses in the range of $10-140 \mu \mathrm{m}$. (c) Experimental data and theoretical curve of the dependence of internal resistance on the thickness of the electrodes. (d) The GCD curves of $100 \mathrm{~mA}$ for the SC models

$$
R_{\text {in }}(h)=r_{0}+\left(\rho_{i}+\rho_{e}\right) h,
$$

where $\rho_{i}\left(\Omega \cdot \mathrm{cm}^{2} / \mu \mathrm{m}\right)$ is the resistivity of transport channels, $\rho_{e}\left(\Omega \cdot \mathrm{cm}^{2} / \mu \mathrm{m}\right)$ is the resistivity of the energy-storage electrode component, respectively. Here $r_{0}\left(\Omega \cdot \mathrm{cm}^{2}\right)$ is the total resistance of the separator (soaked with electrolyte) and the transition layer between the energy-storage electrode component and metal current collector. The latter is determined from the following equation:

$$
\rho_{i}=\frac{R_{i n}\left(h_{1}\right)-R_{i n}\left(h_{2}\right)}{\left(h_{1}-h_{2}\right)}-\rho_{e},
$$

where $R_{\text {in }}(h)\left(\Omega \cdot \mathrm{cm}^{2}\right)$ is the internal resistance of SC model corresponding to the thickness $h . \rho_{n}\left(\Omega \cdot \mu \mathrm{m} \cdot \mathrm{cm}^{2}\right)$ is the resistivity of nanopores carbon material of the energy-storage electrode component. Therefore, $\rho_{n}$ as the ionic resistance of carbon material placed in a rectangular trapezoid with base area $S=1 \mathrm{~cm}^{2}$ and height $h_{t}=1 \mu \mathrm{m}$ (the base of trapezoid is parallel to the metal collector) can be calculated by the following equation:

$$
\rho_{n}=R_{\text {one }} S h_{t} / N
$$

where $N$ is the number of carbon material pores in the volume of trapezoid, and $R_{\text {one }}(\Omega)$ is the ionic resistance of a single carbon material pore, respectively. According to the most reports and our BET results, in the course of charging for the SC device with the organic electrolyte, most of electric charge can be accumulated only in the mesopores with diameters in the range of $2-3 \mathrm{~nm}$. Therefore, only these pores can keep the charges and release them back at SC discharging process. In fact, when charges travel from a transport channel to a pore whose size is comparable to that of ion, these pores will lose their solvation sheath ${ }^{14,15}$ and lead to greatly decrease of diffusion coefficient by several orders of magnitude ${ }^{16}$. Therefore, resistivity resulted from these pores with small diameter can exceed that of a transport channel by several orders of magnitude, and the total resistance of all the micropores can be inversely proportional to the number of those participating in process of discharging.

Based on the above discuss, to further determine $\rho_{n}$, another model assumption should be made. It is supposed that the ionic component of $R_{i n}(h)$ within a certain range of $h$ values can be determined by that of the carbon material. Thus, the ionic component of $R_{i n}(h)$ with two different values $h_{1}$ and $h_{2}\left(h_{1}<h_{2}\right)$ can be calculated within the above range by using Eqs (6) and (7) given below. According to the equivalent circuit of the 

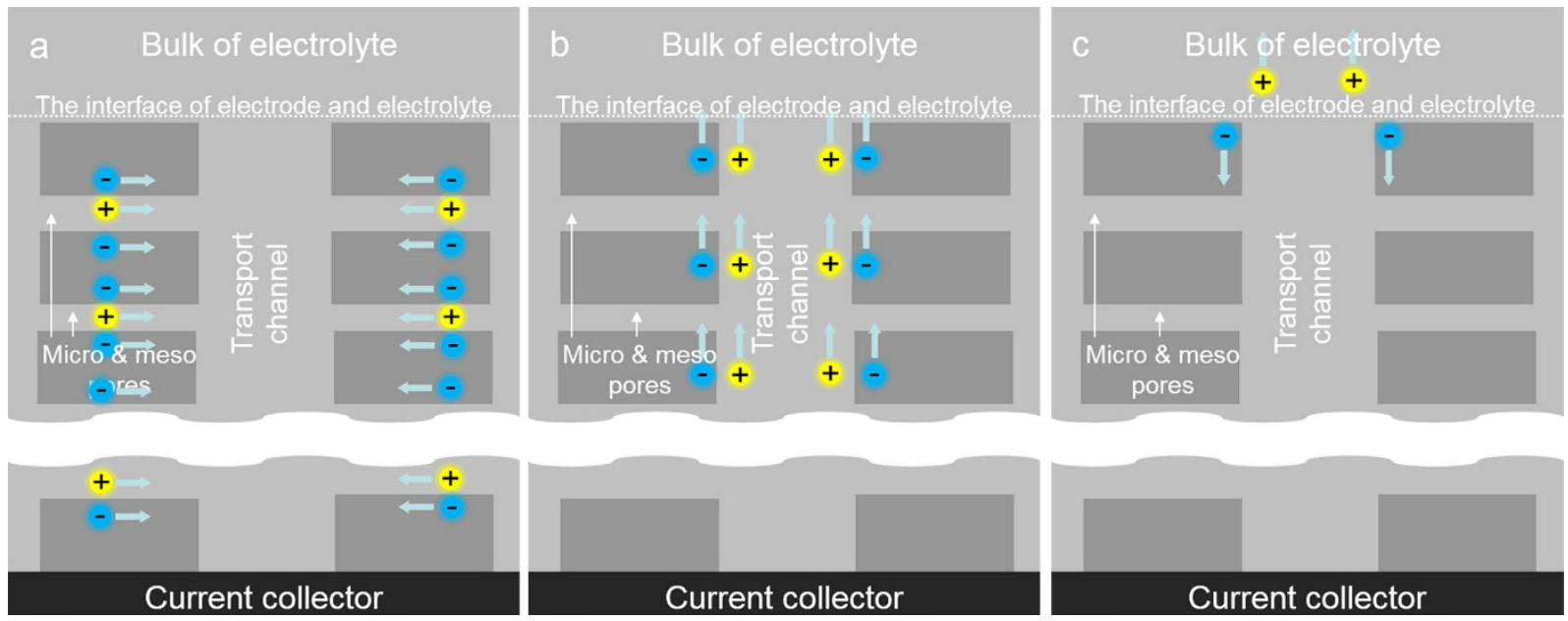

Figure 3. The schematic diagram of the desorbed ions' diffusion.

energy-storage electrode component, the ionic component of internal resistance $r_{\text {ion }}(h)\left(\Omega \cdot \mathrm{cm}^{2}\right)$ can be obtained from Eq. (5):

$$
\begin{aligned}
r_{i o n}(h) & =R_{\text {in }}(h)-r_{0} \\
\frac{\rho_{n}}{h_{1}} & =r_{i o n}\left(h_{1}\right) \\
\frac{\rho_{n}}{h_{2}} & =r_{\text {ion }}\left(h_{2}\right)
\end{aligned}
$$

The validity of our assumption can be verified by the experimental results presented in Fig. $2 c$. In our case, when $h_{1}=14.7 \mu \mathrm{m}$ and $h_{2}=23.5 \mu \mathrm{m}, r_{i o n}(h)=0.81 \Omega \times \mathrm{cm}^{2}$ and, $r_{i o n}(h)=0.52 \Omega \times \mathrm{cm}^{2}$ can be obtained, respectively. Therefore, $\rho_{n}$ can be calculated from the following expression:

$$
\rho_{n}=r_{\text {ion }}\left(h_{1}\right) h_{1}
$$

From our model, it is possible to estimate the internal resistance values dependence of energy-storage component thickness $h^{*}$. The minimal can be found at the cross point of the descending branch (described as $\left.R_{i n}(h)=\frac{\rho_{n}}{h}+r_{0}\right)$ and ascending branch (described as $\left.R_{i n}(h)=r_{0}+\left(\rho_{i}+\rho_{e}\right) h\right)$. The $h^{*}$ value can be calculated as

$$
h^{*}=\sqrt{\frac{\rho_{n}}{\rho_{e}+\rho_{i}}}
$$

Eq. (9) determines thickness of the energy-storage electrode component corresponding to the minimal of SC internal resistance. Within the framework of the model considered, the equation is as follows, describing how the SC internal resistance depends on electrode thickness:

$$
R_{i n}(h)=r_{0}+\frac{\rho_{n}}{h} \theta\left(h^{*}-h\right)+\left(\rho_{e}+\rho_{i}\right) h \theta\left(h-h^{*}\right),
$$

where $\theta(x)$ is the Heaviside step function whose value is 0 if $x<0,0.5$ if $x=0$ and 1 otherwise ${ }^{17}$. The parameters $\rho_{e}+\rho_{i}, r_{0}$ and $\rho_{n}$ that characterize the considered electrochemical system can be calculated based on the experimental data with Eqs (2), (3) and (8). To determine the parameters $r_{0}$ and $\rho_{e}+\rho_{i}$, the points lying along the straight portion of the experimental curve $R_{i n}(h)$ are chosen. In the set of experiments, using the values of SC internal resistance corresponding to the electrode thickness values with $133 \mu \mathrm{m}, 118.2 \mu \mathrm{m}, 88.2 \mu \mathrm{m}$ and $61.2 \mu \mathrm{m}$, respectively, $r_{0}=0.68 \Omega \cdot \mathrm{cm}^{2}$ and $\rho_{e}+\rho_{i}=0.0043 \Omega \cdot \mathrm{cm}^{2} / \mu \mathrm{m}$ can be obtained.

The parameter $\rho_{n}$ can be determined by using these points lying along the experimental $R_{i n}(h)$ curve for which the product $r_{\text {ion }} h$ remains the same. From Table 1, these requirements can be met with the electrodes thickness of $h_{1}=14.7 \mu \mathrm{m} \quad\left(r_{\text {ion }}(h)=0.81 \Omega \times \mathrm{cm}^{2}\right), \quad h_{2}=23.5 \mu \mathrm{m} \quad\left(r_{\text {ion }}(h)=0.52 \Omega \times \mathrm{cm}^{2}\right) \quad$ and $31.3 \mu \mathrm{m}$, $\left(r_{\text {ion }}(h)=0.39 \Omega \times \mathrm{cm}^{2}\right)$ thick, respectively. For SC models with such electrodes, the products $r_{\text {ion }} h$ are in good agreement with the experimental results within the experimental error, indicating the validity of the theoretical model proposed. The mean value of $\rho_{n}$ calculated using the experimental data for the above points is $12.13 \Omega \cdot \mu \mathrm{m} \cdot \mathrm{cm}^{2}$. Using the above $\rho_{e}+$ and $\rho_{n}$ values, the optimized electrode thickness is $h^{*}=53.1 \mu \mathrm{m}$ corresponding to the smallest $\mathrm{R}_{\text {in }}$ value. 


\begin{tabular}{|l|c|c|c|c|c|c|c|c|c|}
\hline $\boldsymbol{h}$ & $\boldsymbol{R}_{\boldsymbol{e}}$ & $\boldsymbol{R}_{\text {in }}$ & $\boldsymbol{r}_{\text {ion }}$ & $\boldsymbol{r}_{\text {ion }} \boldsymbol{h}$ & $\boldsymbol{R}_{\text {in }}$ (theor) & $\boldsymbol{R}_{\text {ion }}$ (theor), & \multicolumn{3}{|c|}{$\mathbf{C}$} \\
\hline $\boldsymbol{\mu m}$ & $\mathbf{\Omega} \cdot \mathbf{c m}^{\mathbf{2}}$ & $\mathbf{\Omega} \cdot \mathbf{c m}^{\mathbf{2}}$ & $\mathbf{\Omega} \cdot \mathbf{c m}^{\mathbf{2}}$ & $\mathbf{\Omega} \cdot \boldsymbol{\mu \mathbf { m } \cdot \mathbf { c m } ^ { \mathbf { 2 } }}$ & $\mathbf{\Omega} \cdot \mathbf{c m}^{\mathbf{2}}$ & $\boldsymbol{\Omega} \cdot \mathbf{c m}^{\mathbf{2}}$ & $\mathbf{F} \cdot \mathbf{c m}^{-\mathbf{3}}$ & $\mathbf{F} \cdot \mathbf{c m}^{-2}$ & $\mathbf{F} \cdot \mathbf{g}^{-1}$ \\
\hline 14.7 & 0.271 & 1.49 & 0.81 & 11.95 & 1.53 & 0.85 & 31.9 & 0.05 & 72.0 \\
\hline 23.5 & 0.325 & 1.20 & 0.52 & 12.30 & 1.21 & 0.53 & 42.6 & 0.10 & 101.7 \\
\hline 31.3 & 0.312 & 1.06 & 0.39 & 12.14 & 1.08 & 0.40 & 46.1 & 0.14 & 111.2 \\
\hline 43.2 & 0.357 & 0.99 & 0.31 & 13.56 & 0.97 & 0.29 & 48.3 & 0.21 & 115.3 \\
\hline 54.3 & 0.385 & 0.92 & 0.22 & 12.13 & 0.92 & 0.23 & 48.0 & 0.26 & 117.4 \\
\hline 61.1 & 0.415 & 0.94 & - & - & 0.94 & - & 50.0 & 0.31 & 120.9 \\
\hline 88.2 & 0.438 & 1.06 & - & - & 1.06 & - & 50.8 & 0.45 & 122.5 \\
\hline 118.2 & 0.505 & 1.17 & - & - & 1.18 & - & 50.6 & 0.60 & 122.1 \\
\hline 133 & 0.559 & 1.26 & - & - & 1.25 & - & 47.0 & 0.63 & 113.6 \\
\hline
\end{tabular}

Table 1. The model parameters, results of theoretical calculations and experimental data obtained for the SC models studied, respectively.

\section{Conclusions}

Based on the experimental studies as well as the correlation between the data obtained and calculated parameters of the prepared SC electrochemical system, the conclusions can be obtained as following:

1. It is found that the dependence of SC internal resistance on the activated carbon-based electrode thickness is nonlinear.

2. A theoretical model is proposed to explain the nonlinear dependence of the internal resistance and electrode thickness. Obviously, the calculations are in good agreement with the experimental results within the experimental error.

3. When the optimized electrode thickness is $53.1 \mu \mathrm{m}$, the SC internal resistance with the minimum value is $12.13 \Omega \cdot \mu \mathrm{m} \cdot \mathrm{cm}^{2}$. The advanced approach can be promising for prediction of parameters of composite materials for SC with high specific power.

\section{References}

1. Sharma, P. \& Bhatti, T. S. A review on electrochemical double-layer capacitors. ENERG CONVERS MAN Energ. Convers. Man. 51, 2901-2912 (2010).

2. Conway, B. E. Electrochemical supercapacitors: Scientific Fundamentals and Technological Applications. Kluwer Academic/Plenum Publishers (1999).

3. Burke, A. Ultracapacitor technologies and application in hybrid and electric vehicles. Int. J. Energ. Res. 34, 133-151 (2010).

4. Lockett, V., Sedev, R., Ralston, J., Horne, M. \& Rodopoulos, T. Differential capacitance of the electrical double layer in imidazoliumbased ionic liquids: Influence of potential, cation size, and temperature. J. Phys. Chem. C 112, 7486-7495 (2008).

5. Manabu, I., Shigek, O., Kenich, M., Kouk, O. \& Masanor, T. Polarized electrode for electric double-layer condenser, and electric double-layer condenser manufactured using the same, and process for manufacturing electrode sheet for electric double-layer condenser, and laminating apparatus: U.S. Patent No. 6831826 (2004).

6. Maletin, Y., Strizhakova, N., Kozachkov, S., Mironova, A. \& Podmogilny, S. Supercapacitor and a method of manufacturing such a supercapacitor: U.S. Patent No. 6697249 (2004).

7. Cui, J. et al. Prolifera-Green-Tide as Sustainable Source for Carbonaceous Aerogels with Hierarchical Pore to Achieve Multiple Energy Storage. Adv. Funct. Mater. 26, 8487-8495 (2016).

8. Maletin, E. Method of fabricating electrodes with low contact resistance for batteries and double-layer capacitors:. U.S. Patent Application No. 11/986013 (2007).

9. Kötz, R. \& Carlen, M. Principles and applications of electrochemical capacitors. Electrochim. Acta 45, 2483-2498 (2000).

10. Yoon, S., Jang, J. H., Ka, B. H. \& Oh, S. M. Complex capacitance analysis on rate capability of electric-double layer capacitor (EDLC) electrodes of different thickness. Electrochim. Acta 50, 2255-2262 (2005).

11. Basirico, L. \& Lanzara, G. Moving towards high-power, high-frequency and low-resistance CNT supercapacitors by tuning the CNT length, axial deformation and contact resistance. Nanotechnology 23, 30 (2012).

12. Lust, E., Janes, A., Parn, T. \& Nigu, P. Influence of nanoporous carbon electrode thickness on the electrochemical characteristics of a nanoporous carbon|tetraethylammonium tetrafluoroborate in acetonitrile solution interface. J. Solid State Electr. 8, 224-237 (2004).

13. Maletin, Y. et al. Matching the nanoporous carbon electrodes and organic electrolytes in double layer capacitors. Appl. Phys. AMater. 82, 653-657 (2006).

14. Everett, D. H. Manual of symbols and terminology for physicochemical quantities and units, appendix II: Definitions, terminology and symbols in colloid and surface chemistry. Pure Appl. Chem. 31, 577-638 (1972).

15. Chmiola, J., Largeot, C., Taberna, P.-L., Simon, P. \& Gogotsi, Y. Desolvation of ions in subnanometer pores and its effect on capacitance and double-layer theory. Angew. Chem.-Int. Edit. 47, 3392-3395 (2008).

16. Zuleta, M., Bursell, M., Bjornbom, P. \& Lundblad, A. Determination of the effective diffusion coefficient of nanoporous carbon by means of a single particle microelectrode technique. J. Electroanal. Electr. Chem. 549, 101-108 (2003).

17. Abramowitz, M. \& Stegun, I. A. Handbook of mathematical functions: with formulas, graphs, and mathematical tables. Courier Corporation, 55 (1964).

\section{Acknowledgements}

This work was supported by the Natural Science Foundation of China (Nos 21571080 and 10604020), Natural Science Foundation of Jilin province, China. Grant Number: 20170101193JC. 


\section{Author Contributions}

Q. Guo and W. Han conceived and directed the experiments. X. L. Liu and X. Dai performed the experiments. G. Wei, V. Izotov, N. Klyui and D. Havrykov co-wrote the manuscript. Y. Xi, and M. Pang participated in analyzing the structure and electrochemical properties of the samples. All authors discussed the results and helped with the preparation of the final manuscript.

\section{Additional Information}

Competing Interests: The authors declare no competing financial interests.

How to cite this article: Liu, X. et al. Experimental and theoretical studies of nonlinear dependence of the internal resistance and electrode thickness for high performance supercapacitor. Sci. Rep. 7, 45934; doi: 10.1038/ srep45934 (2017).

Publisher's note: Springer Nature remains neutral with regard to jurisdictional claims in published maps and institutional affiliations.

(c) (i) This work is licensed under a Creative Commons Attribution 4.0 International License. The images or other third party material in this article are included in the article's Creative Commons license, unless indicated otherwise in the credit line; if the material is not included under the Creative Commons license, users will need to obtain permission from the license holder to reproduce the material. To view a copy of this license, visit http://creativecommons.org/licenses/by/4.0/

(C) The Author(s) 2017 